\title{
Posterior Reversible Encephalopathy Syndrome after Lenvatinib Therapy in a Patient with Anaplastic Thyroid Carcinoma
}

\author{
Yoko Osawa ${ }^{1}$, Rikako Gozawa ${ }^{1}$, Keisuke Koyama ${ }^{1}$, Takeo Nakayama ${ }^{2}$, \\ Tadashi Sagoh ${ }^{3}$ and Hiroshi Sunaga ${ }^{1}$
}

\begin{abstract}
:
Posterior reversible encephalopathy syndrome (PRES) is a rare reversible neurological syndrome that causes subcortical vasogenic brain edema and which is associated with the use of target-specific agents. Lenvatinib is a target-specific agent that was recently approved for inoperable thyroid cancer. We herein describe the case of a 66-year-old woman with anaplastic thyroid cancer (ATC) who was treated with lenvatinib and who subsequently developed PRES. The clinical and radiological findings improved after suspending therapy for 1 week, and there was no recurrence with intermittent lower-dose lenvatinib treatment. Lenvatinib may prolong survival in patients with ATC and can be administered intermittently, even after PRES onset.
\end{abstract}

Key words: anaplastic thyroid carcinoma, lenvatinib, posterior reversible encephalopathy syndrome, tyrosine kinase inhibitor

(Intern Med 57: 1015-1019, 2018)

(DOI: 10.2169/internalmedicine.9593-17)

\section{Introduction}

Anaplastic thyroid cancer (ATC) represents approximately $2 \%$ of all thyroid tumors; the outcome is almost always fatal. The treatment of ATC has not been standardized as most patients die within 6 months of the diagnosis, primarily due to asphyxiation caused by local tumor invasion (1-3). Lenvatinib is a tyrosine kinase inhibitor (TKI) that inhibits the vascular endothelial growth factor receptors and plateletderived growth factor receptors, leading to the inhibition of tumor angiogenesis $(4,5)$. In 2015, lenvatinib was approved for the treatment of inoperable thyroid cancer in Japan. Lenvatinib therapy can prolong the survival of patients with ATC for 1 year or more (6). Lenvatinib therapy is associated mild side effects; however, adverse events including hypertension, bleeding, arterial thrombosis embolism, vein thrombus embolism, hepatopathy, nephropathy, gastrointestinal perforation and fistulation, posterior reversible encephalopathy syndrome, cardiac disorder, hand-foot syndrome, infec- tious disease, myelosuppression, hypocalcemia, delayed wound healing, diarrhea, and fatigue have been reported (7).

Posterior reversible encephalopathy syndrome (PRES) was first described by Hinchey et al. in 1996; PRES is a reversible clinical-radiological syndrome that is characterized by the acute onset of headache, nausea, dizziness, changes in consciousness, convulsions, transient visual disturbances such as cortical blindness, and white matter edema mainly localized to the occipital-parietal lobe (8). PRES can be caused by hypertension, immunosuppressant use, exposure to cytotoxic agents, and the use of molecular target-specific agents; however, the exact cause of PRES is unknown (9). The present report describes the case of a patient with ATC and papillary thyroid cancer who received a total thyroidectomy and right neck dissection followed by lenvatinib treatment for inoperable ATC and subsequently developed PRES.

\section{Case Report}

The patient, a 66-year-old woman, provided her written

${ }^{1}$ Department of Otorhinolaryngology, Fukui Red Cross Hospital, Japan, ${ }^{2}$ Department of Neurology, Fukui Red Cross Hospital, Japan and ${ }^{3}$ Department of Radiology, Fukui Red Cross Hospital, Japan

Received: May 29, 2017; Accepted: July 27, 2017; Advance Publication by J-STAGE: December 8, 2017

Correspondence to Dr. Yoko Osawa, oosawa@ fukui-med.jrc.or.jp 


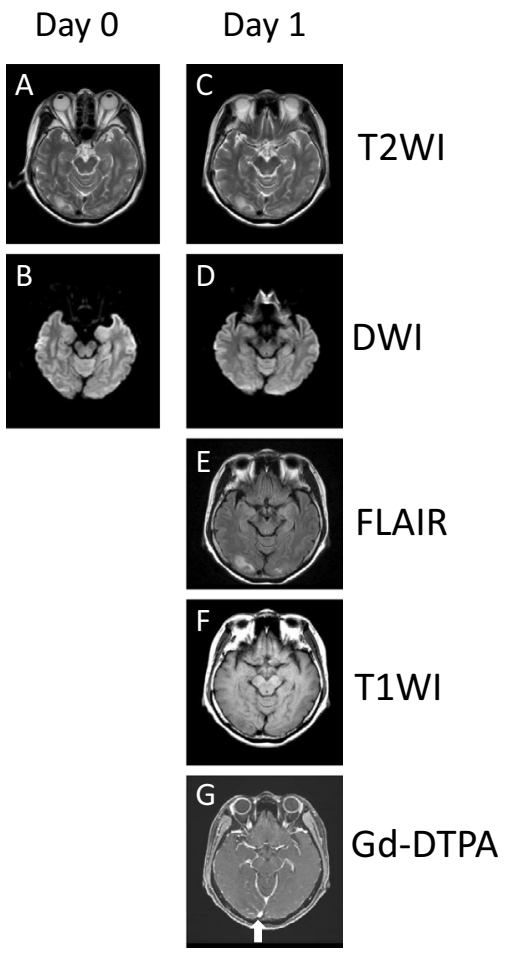

Figure 1. Axial T2-weighted and fluid-attenuated inversion recovery images showing bilateral (right $>$ left) areas of increased signal intensity in the white matter of the occipital lobes on days 0 (A) and 1 (C, E) after the onset of the visual defect. There were no significant findings on T1-weighted $(F)$ or diffusion-weighted imaging $(B, D)$ or in other white matter areas (data not shown). There was no evidence of enhancing lesions in the area of high signal intensity on T2-weighted imaging, and the dural sinus was patent in the whole brain on gadolinium diethylenetriamine pentaacetic acid contrast-enhanced imaging (G, white arrow). T2WI: T2-weighted imaging, DWI: diffusion weighted imaging, FLAIR: fluid-attenuated inversion-recovery imaging, T1WI: T1-weighted imaging, Gd-DTPA: gadolinium diethylenetriamine pentaacetic acid

informed consent for treatment. She had undergone a total thyroidectomy and right conservative neck dissection to treat bilateral thyroid cancer with right neck lymph node metastasis, right papillary thyroid cancer (pT3 N1b M0), and left ATC (pT4b N0 M0). Preoperative fine needle aspiration biopsy identified suspicious papillary cancer cells (class V) in the right thyroid lobe and suspicious carcinoma cells of unknown histological type (class V) in the left thyroid lobe. The ATC had infiltrated the constrictor pharyngeal and cervical esophagus muscles; however, the patient elected not to undergo the removal of her larynx; thus, it was not possible to completely resect the ATC. Her relevant medical history included, type II diabetes mellitus, hypercholesterolemia, angina, and transient cerebral ischemia. Her blood pressure was controlled to within the normal range (130-140/70-80 $\mathrm{mmHg}$ ) with antihypertensive agents. The patient's HbAlc level was $6.3 \%$ with the self-administration of subcutaneous insulin. Her performance status was 0 and a physical examination revealed no relevant physical abnormalities.
Nine days after the operation, we confirmed that all skin wounds were closed and subsequently initiated lenvatinib therapy $(24 \mathrm{mg} /$ day $)$ to treat the remaining cancer of the constrictor pharyngeal and cervical esophagus muscles. Initially, the patient did not experience any adverse events; however, 19 days after the initiation of treatment, she presented with hypertension. The dose of lenvatinib was reduced $(20 \mathrm{mg} /$ day $)$ and an antihypertensive drug was added to her treatment regimen.

Twenty-nine days after the initiation of lenvatinib treatment (10 days after the dose reduction), the patient noticed a defect in the upper left side of her visual field while she was self-administering insulin. She went to the nearest ophthalmological clinic, but the ophthalmologist suggested that her symptoms were related to a central nervous system disorder. On the same day, the patient consulted with our emergency department. Cranial computed tomography (CT), magnetic resonance imaging (MRI), and magnetic resonance angiography (MRA) were performed. No evidence of acute cerebral bleeding or infarction was identified on CT, MRI, or MRA images. The next day (30 days after the initiation of lenvatinib treatment), she visited our department accompanied by her husband and son because of the appearance of additional symptoms, including forgetfulness, incoherent speech, and restlessness mimicking dementia as well as difficulty in self-administering medications. Her blood pressure was elevated $(172 / 83 \mathrm{mmHg})$ and her neurological status had deteriorated. The patient had a Glasgow Coma Scale (GCS) score of 12 (E4, V3, M5). The patient's heart rate was 81 beats per minute and the electrocardiography findings were within the normal limits. A laboratory analysis revealed that her blood sugar level was $164 \mathrm{mg} / \mathrm{dL}$ and her HbA1c level was 5.8\%. We consulted with neurologists who noted that the patient's previous MRI scan showed abnormal findings. Upon re-examination, T2-weighted and fluidattenuated inversion recovery (FLAIR) MRI scans that had been obtained on the previous day and the same day revealed bilateral areas of increased signal intensity in the white matter of the occipital lobes (Fig. 1A, C and E). PRES was suspected based on the MRI findings. There were no notable findings on T1-weighted or diffusion-weighted images (Fig. 1B, F and D) or in the other white matter areas (data not shown). MRA performed on the previous day did not indicate the presence of lesions of cerebral angiostenosis or cerebral angiectasis, or any regional differences in the cerebral blood flow (data not shown). There was no abnormal stenosis of the main arteries, including stenosis of the large arteries in the trunk of the body on preoperative enhanced CT images (data not shown). Moreover, there were no enhancing lesions in the area that showed high intensity on T2-weighted imaging and the dural sinus was patent in the whole brain on gadolinium diethylenetriamine pentaacetic acid contrast-enhanced imaging (Fig. 1G).

The patient was admitted to our hospital and treated with antihypertensive agents including an angiotensin II receptor antagonist and a calcium antagonist. Type-II diabetes melli- 


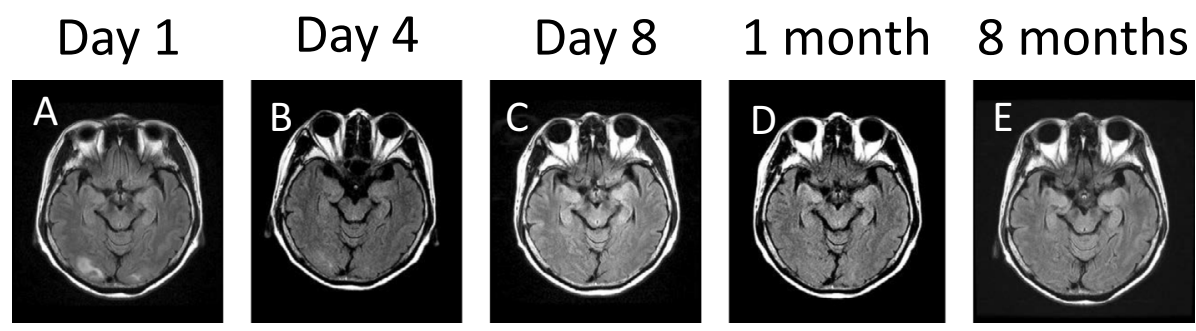

\begin{abstract}
Figure 2. Follow-up fluid-attenuated inversion recovery imaging showing the reduction of edema (decreased signal intensity) in the white matter of the occipital lobes on day 4 (B) after the onset of the visual defect in comparison to day 1 (A). Fluid-attenuated inversion recovery imaging shows the progressive reduction of edema and normalization by day 8 after the onset of the visual defect (C); normal findings were observed 1 month (D) and 8 months (E) later, during intermittent lenvatinib treatment.
\end{abstract}

tus was treated with the injection of rapid-acting insulin, which had previously been suspended due to the patient's impaired consciousness. The next day, the patient recovered from her confused mental state, but remained disoriented and continued to complain of a defect in the upper left side of her visual field. On day 4 after the onset of the visual defect, the visual disturbance resolved and FLAIR images showed a reduction of edema (decreased signal intensity) in the white matter of the occipital lobes (Fig. 2B). On day 8 after the onset of the visual defect, the patient was found to have completely recovered from her consciousness disturbance (GCS score: 15), and FLAIR images showed that areas of high signal intensity in the occipital lobes had completely resolved (Fig. 2C).

In accordance with the LENVIMA guidebook, which contains information about the proper use of lenvatinib, we reduced the patient's dose of lenvatinib to $14 \mathrm{mg} /$ day at 8 days after the onset of the visual defect. After 13 days of treatment with lenvatinib at a dose of $14 \mathrm{mg} /$ day (day 21 after the onset of the visual defect), the patient presented with hypertension and hepatopathy with increased serum levels of alanine aminotransferase (ALT) and aspartate aminotransferase (AST) and increased urinary levels of protein. Lenvatinib therapy was discontinued; 4 days later, adverse effects related to lenvatinib treatment completely disappeared and the patient resumed lenvatinib therapy at a lower dose (10 mg/day). Follow-up cranial MRI did not reveal any areas of high signal intensity (Fig. 2D). Nine days after resuming lenvatinib therapy (day 34 after the onset of the visual defect), the patient again presented with hypertension, hepatopathy, increased serum levels of ALT/AST, and increased urinary levels of protein. Lenvatinib was again discontinued and the adverse effects disappeared after 7 days. The patient resumed lenvatinib therapy at a dose of $8 \mathrm{mg} /$ day. The patient eventually died due to rupture of the left common carotid artery, 12 months after the first medical examination (9 months after the diagnosis of ATC was established); she did not experience any other episodes impaired consciousness. Cranial MRI scans obtained 7 days before her death ( 7 months after the onset of the visual defect), revealed no areas of high signal intensity, confirming the ab- sence of PRES (Fig. 2E).

\section{Discussion}

We reported the case of a patient with ATC who developed PRES after treatment with lenvatinib. Our case shows the outcome of repeated treatment with decreasing doses of lenvatinib after the onset of PRES. No other study to date has described the outcome of repeated treatment with a TKI after the appearance of PRES.

PRES-associated visual disturbances can become obvious as the disease evolves, whereas hypertension typically presents early after the onset of PRES, although some cases involving normal blood pressure have been reported $(9,10)$. The lesions of PRES are normally localized to the posterior regions of the brain and can affect both the white and grey matter; however, these lesions are usually reversible (9). The development of MRI technology has made it easier to diagnose PRES. In the present case, our patient experienced visual disturbance and hypertension in the early stage of PRES and later developed mental dysfunction with areas of increased signal intensity in the white matter of the bilateral occipital lobes on T2-weighted and FLAIR MRI. A 7-day interruption in lenvatinib treatment $(20 \mathrm{mg} /$ day $)$ allowed the patient to recover from the visual and consciousness disturbances; during this time, the white matter lesions completely disappeared. This finding indicated that lenvatinib induced typical PRES in the present case.

The medications that have been associated with PRES primarily include immunosuppressant agents and molecular target-specific agents, including a number of TKIs (cediranib, sunitinib, sorafenib, pazopanib, and regorafenib) (11-18). Lenvatinib is a TKI that has been approved for the treatment of inoperable thyroid cancer, including ATC, in Japan. According to the LENVIMA guidebook, no Japanese patients developed PRES in phase II and phase III trials of lenvatinib treatment. In a phase III trial from another country, only 1 of 261 patients developed PRES after lenvatinib treatment (7). Thus, the present case represents the first reported case of PRES associated with lenvatinib use in a Japanese patient. 
The concentration of lenvatinib may persist in the body for more than 7 days (19). In the present case, a 7-day interruption in therapy allowed for the resolution of PRES symptoms and MRI findings, suggesting that lenvatinib treatment was the cause of PRES. One study suggested that severe hypertension causing vasogenic edema can also precipitate PRES (20). It is unlikely that PRES was directly caused by severe hypertension - independent of lenvatinib use - in the present case, as the PRES symptoms disappeared during the suspension of lenvatinib treatment and reappeared after its re-initiation, despite the patient's recurrent severe hypertension. Lenvatinib inhibits vascular endothelial growth factor receptors (VEGFR) 1-3 in order to disrupt tumor angiogenesis and thus tumor invasion and metastasis (21). It is known that vascular endothelial growth factor (VEGF) regulates vasomotor tonus and maintains the blood pressure by dilating small arterioles and venules (22). The LENVIMA guidebook reported that $70 \%$ of patients treated with lenvatinib developed hypertension in a phase III trial (7). Thus, it is possible that hypertension related to lenvatinib use contributed to the appearance of PRES.

In the present case, we repeatedly administered lenvatinib to the patient despite severe side effects, including PRES, because we thought that lenvatinib would prolong her survival. It was recently reported that lenvatinib treatment was effective for locally advanced ATC and led to an improved quality of life as well as prolonged survival in Japanese patients $(2,23)$. As previously mentioned, most ATC patients die within 6 months of the diagnosis (1-3). The patient in our case lived for 8 months after the postoperative diagnosis of ATC, while receiving lenvatinib treatment. Notably, PRES did not recur in the present case; this is consistent with a previous report, which noted that recurrent PRES attacks are rare (24). Taken together, these data suggest that intermittent lenvatinib treatment can prolong survival in patients with ATC.

In conclusion, this is the first report about a case of PRES induced by lenvatinib treatment for ATC. All of the clinical and radiological findings of PRES disappeared after one week of treatment interruption, and there was no recurrence of PRES, despite repeated treatment with progressively lower doses of lenvatinib. Intermittent lenvatinib treatment may prolong the survival of patients with inoperable ATC.

The authors state that they have no Conflict of Interest (COI).

\section{References}

1. Chiacchio S, Lorenzoni A, Boni G, Rubello D, Elisei R, Mariani G. Anaplastic thyroid cancer: prevalence, diagnosis and treatment. Minerva Endocrinol 33: 341-357, 2008.

2. Sugitani I, Miyauchi A, Sugino K, Okamoto T, Yoshida A, Suzuki S. Prognostic factors and treatment outcomes for anaplastic thyroid carcinoma: ATC Research Consortium of Japan cohort study of 677 patients. World J Surg 36: 1247-1254, 2012.

3. Akaishi J, Sugino K, Kitagawa W, et al. Prognostic factors and treatment outcomes of 100 cases of anaplastic thyroid carcinoma.
Thyroid 21: 1183-1189, 2011.

4. Keizer RJ, Gupta A, MacGillavry MR, et al. A model of hypertension and proteinuria in cancer patients treated with the antiangiogenic drug E7080. J Pharmacokinet Pharmacodyn 37: 347363, 2010.

5. Schlumberger M, Tahara M, Wirth LJ, et al. Lenvatinib versus placebo in radioiodine-refractory thyroid cancer. N Engl J Med 372: 621-630, 2015.

6. Tahara M, Kiyota N, Yamazaki T, et al. Lenvatinib for Anaplastic Thyroid Cancer. Front Oncol 7: 25, 2017.

7. LENVIMA Guidebook; lenvatinib management information for doctors provided by Eisai. [cited 2015 May 1]. Available from: htt p://www.eisai.co.jp

8. Hinchey J, Chaves C, Appignani B, et al. A reversible posterior leukoencephalopathy syndrome. N Engl J Med 334: 494-500, 1996.

9. Fugate JE, Rabinstein AA. Posterior reversible encephalopathy syndrome: clinical and radiological manifestations, pathophysiology, and outstanding questions. Lancet Neurol 14: 914-925, 2015.

10. Li R, Mitchell P, Dowling R, Yan B. Is hypertension predictive of clinical recurrence in posterior reversible encephalopathy syndrome? J Clin Neurosci 20: 248-252, 2013.

11. Glusker P, Recht L, Lane B. Reversible posterior leukoencephalopathy syndrome and bevacizumab. N Engl J Med 354: 980-982, 2006.

12. Ozcan C, Wong SJ, Hari P. Reversible posterior leukoencephalopathy syndrome and bevacizumab. N Engl J Med 354: 980-982, 2006.

13. Dogan E, Aksoy S, Arslan C, Dede DS, Altundag K. Probable sorafenib-induced reversible encephalopathy in a patient with hepatocellular carcinoma. Med Oncol 27: 1436-1437, 2010.

14. Padhy BM, Shanmugam SP, Gupta YK, Goyal A. Reversible posterior leucoencephalopathy syndrome in an elderly male on sunitinib therapy. Br J Clin Pharmacol 71: 777-779, 2011.

15. Palma JA, Gomez-Ibañez A, Martin B, Urrestarazu E, Gil-Bazo I, Pastor MA. Nonconvulsive status epilepticus related to posterior reversible leukoencephalopathy syndrome induced by cetuximab. Neurologist 17: 273-275, 2011.

16. Chelis L, Souftas V, Amarantidis K, et al. Reversible posterior leukoencephalopathy syndrome induced by pazopanib. BMC Cancer 12: 489, 2012.

17. Myint ZW, Sen JM, Watts NL, et al. Reversible posterior leukoencephalopathy syndrome during regorafenib treatment: a case report and literature review of reversible posterior leukoencephalopathy syndrome associated with multikinase inhibitors. Clin Colorectal Cancer 13: 127-130, 2014.

18. Kim CA, Price-Hiller J, Chu QS, et al. Atypical reversible posterior leukoencephalopathy syndrome (RPLS) induced by cediranib in a patient with metastatic rectal cancer. Invest New Drugs 32: 1036-1045, 2014.

19. Kitamura M, Hayashi T, Suzuki C, et al. Successful recovery from a subclavicular ulcer caused by lenvatinib for thyroid cancer: a case report. World J Surg Oncol 15: 24, 2017.

20. Xie C, Jones VT. Reversible posterior leukoencephalopathy syndrome following combinatorial cisplatin and pemetrexed therapy for lung cancer in a normotensive patient: a case report and literature review. Oncol Lett 11: 1512-1516, 2016.

21. Stjepanovic N, Capdevila J. Multikinase inhibitors in the treatment of thyroid cancer: specific role of lenvatinib. Biologics 8: 129-139, 2014.

22. Ancker OV, Wehland M, Bauer J, et al. The adverse effect of hypertension in the treatment of thyroid cancer with multi-kinase inhibitors. Int J Mol Sci 18: E625, 2017.

23. Fukuhara T, Donishi R, Koyama S, et al. Significant amelioration of tracheal stenosis following lenvatinib in a patient who has anaplastic thyroid carcinoma with bronchomediastinal infiltration: 
a case report. Case Rep Oncol 10: 175-181, 2017.

24. Roth C, Ferbert A. Posterior reversible encephalopathy syndrome: long-term follow-up. J Neurol Neurosurg Psychiatry 81: 773-777, 2010 .
The Internal Medicine is an Open Access article distributed under the Creative Commons Attribution-NonCommercial-NoDerivatives 4.0 International License. To view the details of this license, please visit (https://creativecommons.org/licenses/ by-nc-nd/4.0/).

(C) 2018 The Japanese Society of Internal Medicine Intern Med 57: 1015-1019, 2018 